\title{
Bifurcation of a Fractional-order Complex System
}

\author{
Honggang Dang ${ }^{a}$, Xiaoya Yang, XiaoJun Liu \\ School of Mathematics and statistics, Tianshui Normal University, Tianshui, China \\ afly0164@126.com
}

Keywords: Fractional-order system, bifurcation, chaos.

\begin{abstract}
In this paper, bifurcation of a fractional-order complex system is studied. As system parameter is varied, bifurcation of the system is showed by the numerical simulation. The routes leading to chaos including the period-doubling and tangent bifurcations are obtained. The phase portraits for different value of parameters are also given to demonstrate the dynamics of the system.
\end{abstract}

\section{Introduction}

Nowadays, fractional calculus plays an important role in mathematical modeling. It has been applied to physics, engineering, secure communications and so on. Meanwhile, chaotic behavior is also obtained in many fractional-order systems, such as fractional-order Chua circuit, fractional-order Lorenz system, fractional-order Chen system, and so on [1-3]. As research continues, the importance of fractional-order systems with complex variables is realized by many researchers, which can be widely used to describe a variety of physical phenomena, for example, the atomic polarization amplitudes, electric field, population inversion, etc [4-6].

\section{System description}

In [7], a new three-dimensional system was presented, which can be described by the following differential equations

$$
\left\{\begin{array}{l}
\dot{y}_{1}=a\left(y_{2}-y_{1}\right) \\
\dot{y}_{2}=y_{1} y_{3}-y_{2} \\
\dot{y}_{3}=b-y_{1} y_{2}-c y_{3}
\end{array},\right.
$$

where $y=\left(y_{1}, y_{2}, y_{3}\right)^{\mathrm{T}}$ is the state variable vector of the system, $a, b, c$ are parameters. When the parameters $a=5, b=16, c=1$, the system exists an chaotic attractor.

In here, the state variables of system (1) are defined in the complex field, then the corresponding fractional-order system is defined as

$$
\left\{\begin{array}{l}
D^{q} y_{1}=a\left(y_{2}-y_{1}\right) \\
D^{q} y_{2}=y_{1} y_{3}-y_{2} \\
D^{q} y_{3}=b-\frac{1}{2}\left(\bar{y}_{1} y_{2}+y_{1} \bar{y}_{2}\right)-c y_{3}
\end{array},\right.
$$

where $q$ is the order of derivative, $y=\left(y_{1}, y_{2}, y_{3}\right)^{\mathrm{T}}$ is the vector of state variables. $y_{1}=x_{1}+i x_{2}, y_{2}=x_{3}+i x_{4}$ are complex variables, $y_{3}=x_{5}$ is real variable, and $i=\sqrt{-1}$. Then the complex variables in the system are separated into the real and imaginary parts, respectively. According to the linearity of the Caputo differential operator, the system (2) can be rewritten as 


$$
\left\{\begin{array}{l}
D^{q} x_{1}=a\left(x_{3}-x_{1}\right) \\
D^{q} x_{2}=a\left(x_{4}-x_{2}\right) \\
D^{q} x_{3}=x_{1} x_{5}-x_{3} \\
D^{q} x_{4}=x_{2} x_{5}-x_{4} \\
D^{q} x_{5}=b-\left(x_{1} x_{3}+x_{2} x_{4}\right)-c x_{5}
\end{array}\right.
$$

Compared with the system (2), (3) is more convenient for analysis and numerical simulation. By numerical computation, the maximum Lyapunov exponent of the system (3) with $a=5, b=16, c=1, q=0.99$ is $\lambda_{1}=0.071$, which means the system (3) is chaotic. The chaotic attractors on different phase space projections are shown in Fig.1
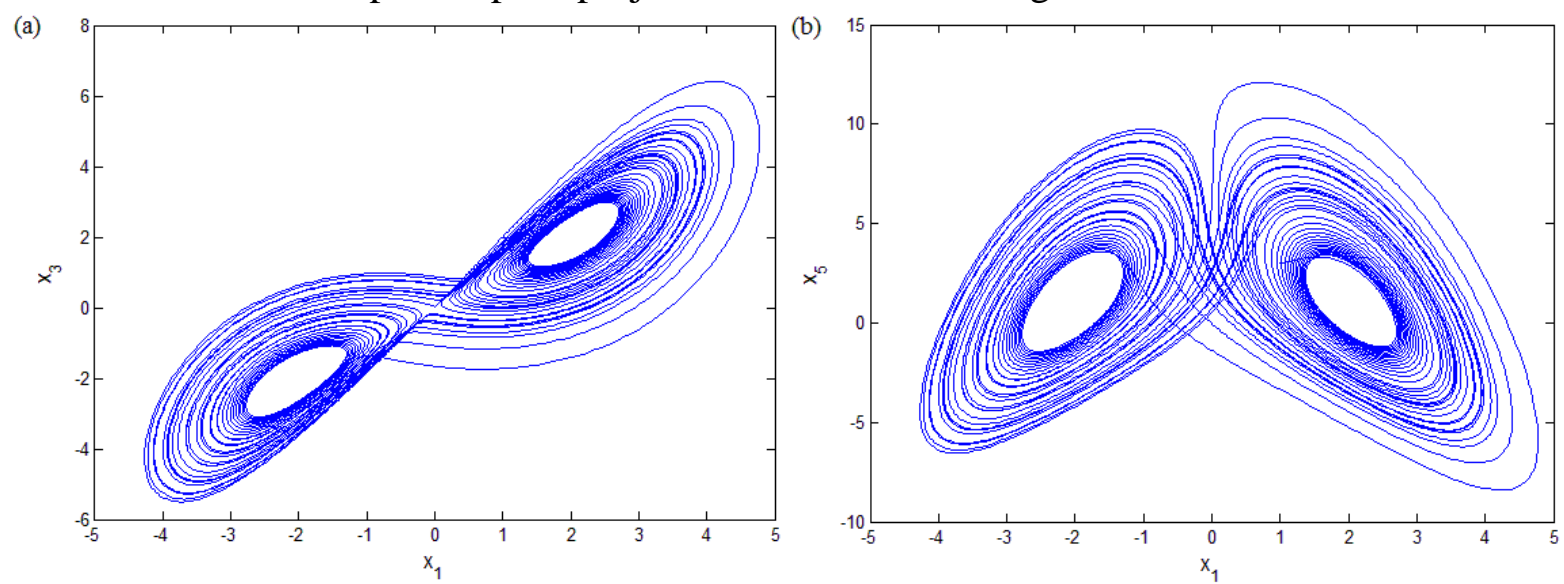

Fig.1. The chaotic attractors of the system (3) projected onto different phase plane. (a) $x_{1}-x_{3}$ phase plane; (b) $x_{1}-x_{5}$ phase plane.

Firstly, the bifurcation of system (3) as the parameter $a$ is varied will be investigated when the order is taken as $q=0.99$, and system parameters $b=16, c=-0.2$. From Fig.2, there is one limit cycle until period-doubling bifurcation occurs when $a=6.45$. When $a \in[1,1.5]$ and $a \in[4,4.5]$, a period-doubling bifurcation can be obtained . The corresponding phase portraits are shown in Fig.3, from which it can be seen that the system (3) exists period-1, period-2 for different values of the parameter $a$.

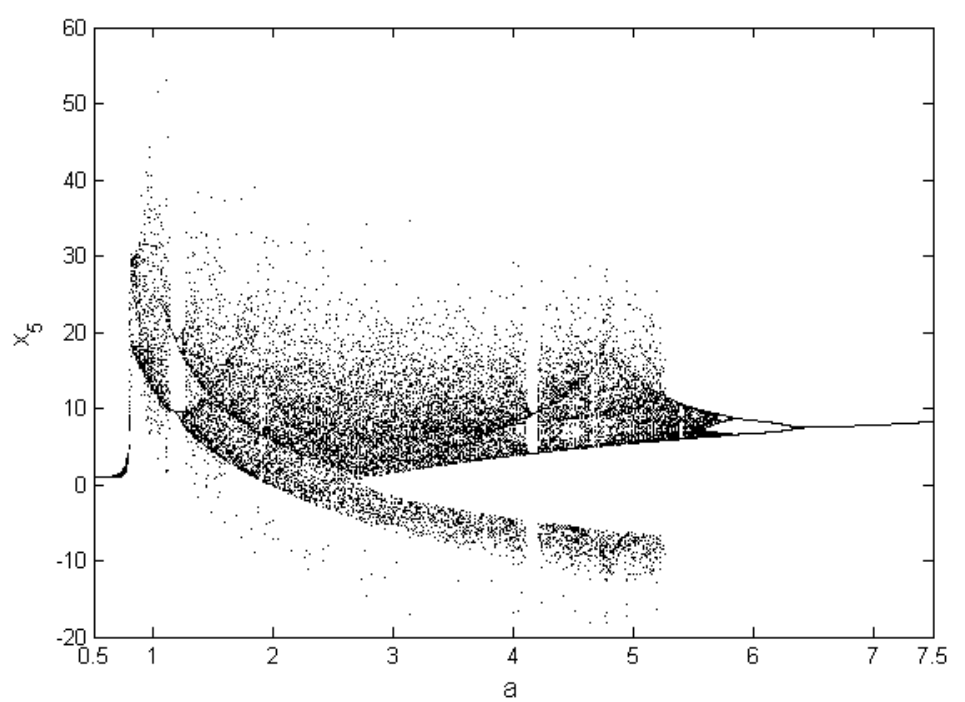

Fig.2. The bifurcation diagram of the system (3) with parameter $a \in[0.5,7.5]$. 

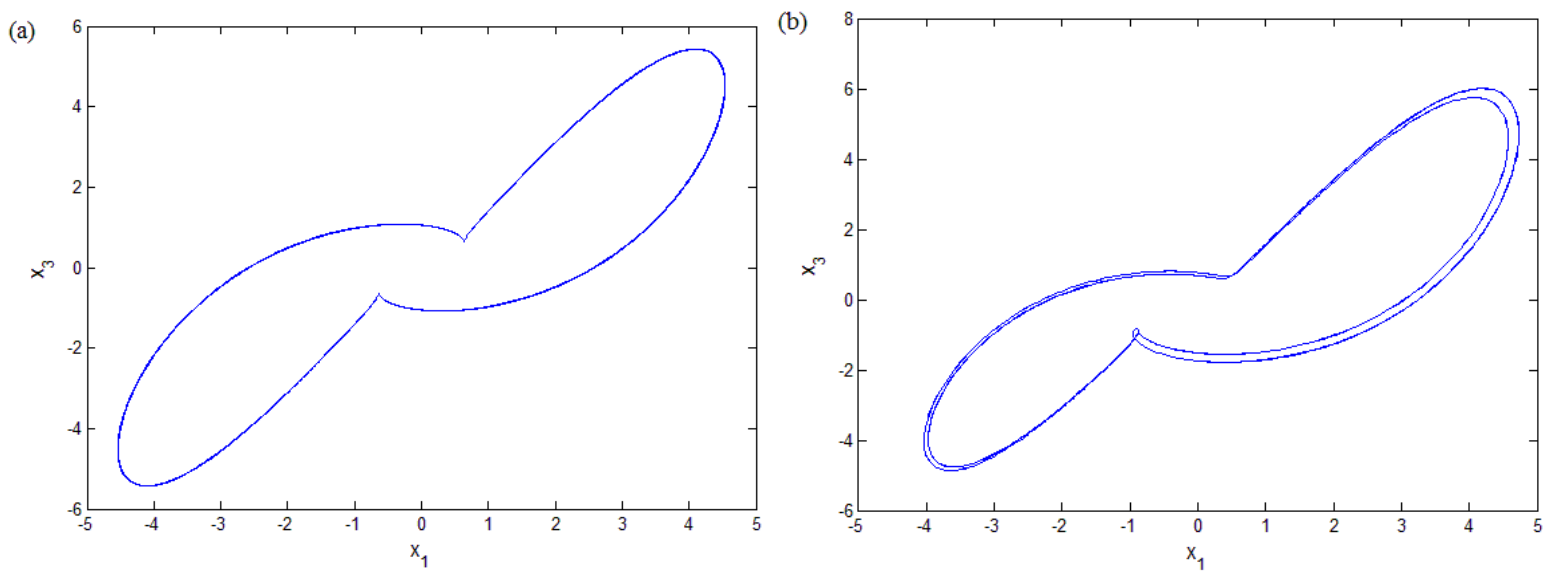

Fig.3. The phase portraits of the system for different values of the parameter $a$ (a) $a=7$; (b) $a=5.8$.

Secondly, the dynamics of system (3) varying the parameter $b$ will be studied when $a=5, c=1$, and $q=0.99$. Via the numerical computation, the bifurcation of the system (3) when the parameter $b \in[10,125]$ is obtained which is depicted in Fig.4. It is clear that with the decrease of the parameter $b$, the route out of chaos for the system is through tangent bifurcation when $b=110.5$. Then two limit cycles coexist until period-doubling bifurcation occurs when $b=105$. By a series of period-doubling bifurcations, the system enters into chaos again.

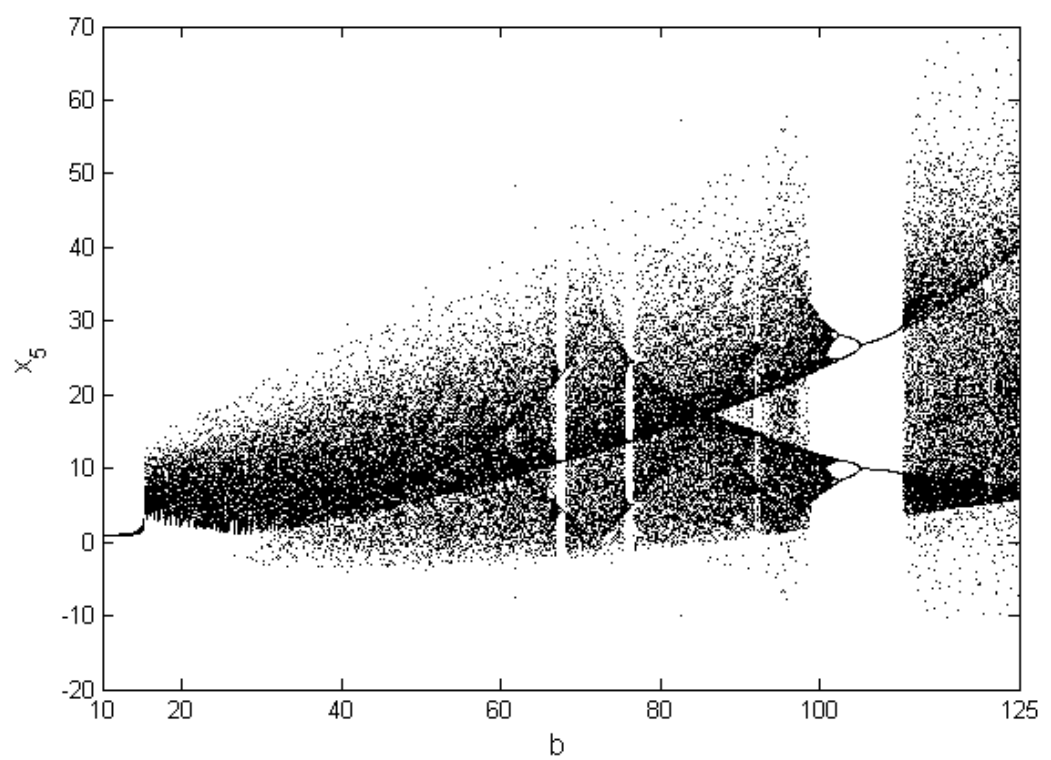

Fig.4. The bifurcation diagram of the system (11) with parameter $b \in[10,125]$.

Thirdly, when the system parameters are taken as $a=5, b=16$, the derivative order $q=0.99$, the bifurcation diagram of the system (3) with parameter $c$ is shown in Fig.5. The tangent bifurcation occurs when $c=0.05$ and the period-doubling bifurcations can be seen with $c \in[0.04,0.09]$. 


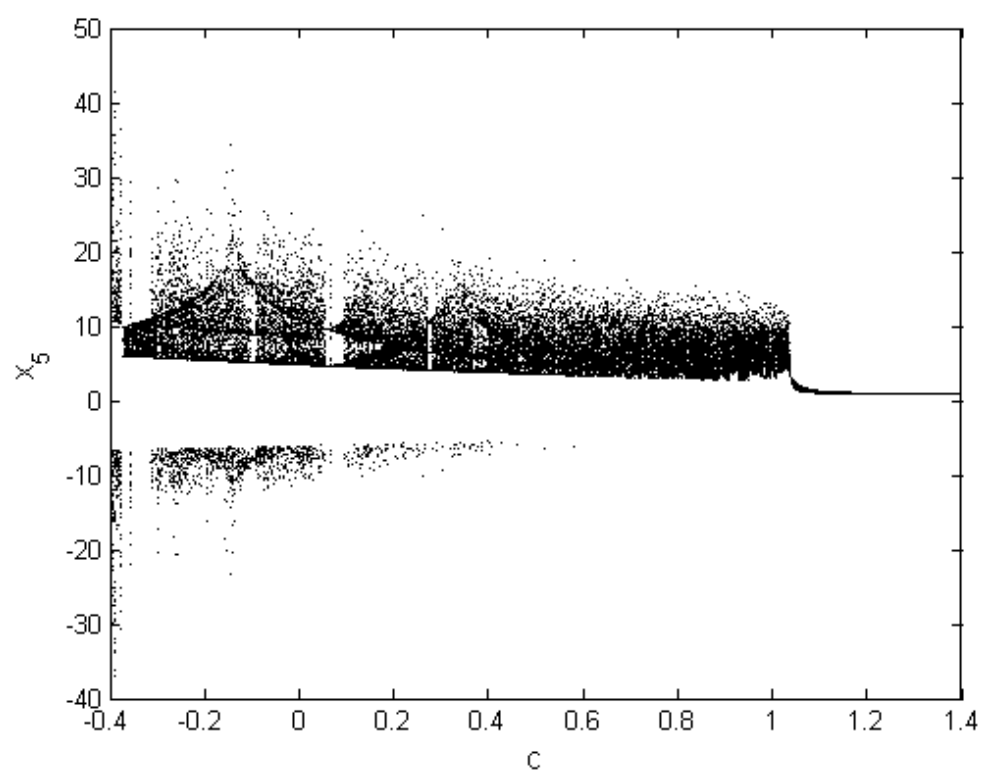

Fig.5. The bifurcation diagram of the system (3) with parameter $c \in[-0.4,1.4]$.

\section{Conclusions}

In this paper, bifurcation of a fractional-order complex system is investigated. As the system parameter is varied, bifurcation of the system is showed by the numerical simulation. The routes leading to chaos including the period-doubling and tangent bifurcations are obtained. The phase portraits for different value of parameters are also given to demonstrate the dynamics of the system.

\section{References}

[1] C.P. Li, W.H Deng, and D. Xu: Chaos synchronization of the Chua system with a fractional order. Physica A, 360(2006): 171-185.

[2] P. Arena, R. Caponetto, L. Fortuna, and D. Porto: Chaos in a fractional order Duffing system. In: Proc ECCTD. Budapest, (1997): 1259-1262.

[3] J.G. Lu, G.R. Chen: A note on the fractional-order Chen system. Chaos Solitons \& Fractals, 27(2006): 685-688.

[4] G. Mahmoud, T. Bountis : The dynamics of systems of complex nonlinear oscillators: a review. Int. J. Bifurcation chaos, 14 (2004): 3821-3846.

[5] M. Moghtadaei and M.H. Golpayegani Complex dynamic behaviors of the complex Lorenz system. Scientia Iranica 19(2012): 733-738.

[6] G. Mahmoud, S. Aly, M. AL-Kashif: Dynamical properties and chaos synchronization of a new chaotic complex system. Nonlinear Dynamics, 51(2008): 171-181.

[7] X.F. Li, Y.D. Chu, J.G. Zhang, and Y.X. Chang: Nonlinear dynamics and circuit implementation for a new autonomous chaotic system. Chaos Solitions \& Fractals, 41(2009): 2360-2370. 